\title{
Psoriatic March, Skin Inflammation and Cardiovascular Events - Two Plaques for one Syndrome
}

José Laerte Boechat ${ }^{1,2}$ (๑)

Universidade Federal Fluminense - Clinical Immunology Unit, ${ }^{1}$ Niterói, RJ - Brazil

Universidade do Porto - Basic and Clinical Immunology Unit, Department of Pathology Porto, ${ }^{2}$ Porto - Portugal

Traditionally, psoriasis has been regarded as a disease affecting only the skin and joints. Unfortunately, this is still the view among many doctors.

In this issue, Campos et al., ${ }^{1}$ published an interesting observational study on cardiovascular (CV) risk factors and risk measurement in patients with psoriatic arthritis, which allow us, in this editorial, to put the spotlight on some points of great interest in this area.

As the understanding of psoriasis has evolved, the perception of the disease pathophysiology changed from an organ-specific hyperkeratotic disorder of keratinocytes to an underlying dysregulation of the immune system mediated by cytokines. These concepts led to a further shift in its classification from a skin disease to a T-cell mediated disease. ${ }^{2}$

In the last years, a number of studies have demonstrated that psoriasis, mainly with systemic involvement (psoriatic arthritis), is associated with a higher prevalence of $\mathrm{CV}$ and metabolic diseases, and the prevalence of $\mathrm{CV}$ risk factors are increased in patients with psoriatic arthritis, including hypertension, diabetes, obesity, dyslipidemia and metabolic syndrome. ${ }^{3}$ The results of the cross-sectional study by Campos et al., ${ }^{1}$ support these associations.

The close association of skin inflammation with $\mathrm{CV}$ disease and metabolic syndrome is a relative new topic in medicine. Inflammatory skin diseases, such as atopic dermatitis and psoriasis, produce a wide range of proinflammatory cytokines and chemokines not only in the lesional skin, but also in the circulation, causing

\section{Keywords}

Psoriasis; Dermatitis,Atopic; Risk Factors; Systemic Inflammatory Response Syndrome; Arthritis, Psoriatic; Inflammation; Comorbidity. systemic inflammation, insulin resistance and endothelial dysfunction, which may lead to atherosclerosis, myocardial infarction and CV comorbidities - the so called psoriatic

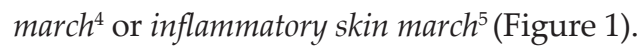

The chronic state of inflammation appears to be a central mechanism underlying the pathophysiology of insulin resistance, visceral adiposity, hypertension and dyslipidemia. ${ }^{6}$ But, what are the molecular mechanisms responsible for the association between psoriasis and cardiovascular comorbidities?

In 2012, Flammer and Ruschitzka ${ }^{7}$ proposed the theory of two plaques for one syndrome. The cytokine profiles of

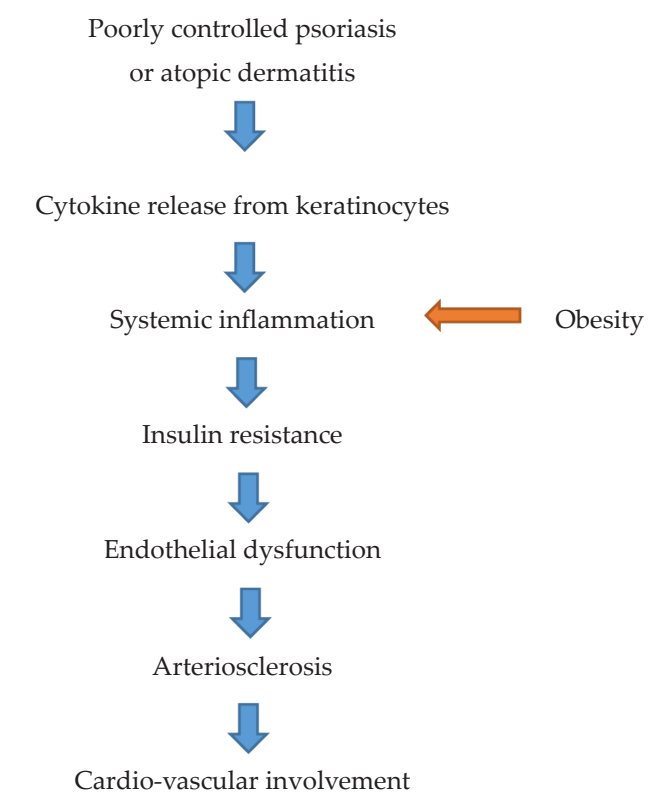

Figure 1 - The concept of inflammatory skin march. Modified from ref $^{4,5}$. 
psoriasis skin lesions and atherosclerosis vascular lesions are very similar, showing increased number of Th1 and Th17 lymphocytes. The Th1 and Th17 cytokine pathways have been shown to be involved in the pathogenesis of both psoriasis and atherosclerosis. The overexpression of Th17 cytokines in patients with psoriasis may mediate vascular inflammation and the development of atherosclerosis and CV comorbidities. Other mechanisms proposed are shared genetic factors, secretion of adipokines, lipoprotein composition and function, angiogenesis, oxidative stress, microparticles and hypercoagulability. ${ }^{8}$

While metabolic comorbidities are still underdiagnosed and undertreated in patients with long-standing psoriasis, the question arises whether earlier and more stringent control of the disease-driving inflammatory processes could potentially prevent the development and worsening of comorbidities. In this context, can we consider psoriasis as an independent risk factor for $\mathrm{CV}$ disease?

Several factors are associated with a higher risk of developing CV disease, including age, high blood pressure, obesity, smoking, stress and family history. Many (if not all) of these risk factors are also present in psoriatic patients. ${ }^{9}$ Metabolic syndrome is more frequent in patients with psoriasis than in general population. Campos et al. ${ }^{1}$ found dyslipidemia in 93\%, hypertension in $46 \%$, obesity in $40 \%$ and diabetes in $33 \%$ of the sample studied (psoriatic arthritis patients aged between 30 to 74 years, without other chronic inflammatory diseases, seen at the rheumatology outpatient clinic of a referral hospital in João Pessoa / Paraiba).

$\mathrm{Su}$ et $a l .,{ }^{10}$ found that the risk of developing severe vascular events is higher when psoriasis acts as a disease amplifier (i.e., when metabolic disorder precedes psoriasis), compared to when it acts as the disease initiator. Reich ${ }^{2}$ proposed a simplified model, based on the excellent paper by Davidovici et al., ${ }^{11}$ that illustrates many potential functional interplays between shared genetic risk factors that drive both psoriasis and obesity, and may promote the development of CV comorbidities (Figure 2).

There are, however, opposing arguments to this model, and the debate so far has been whether there is a causal relationship between psoriasis and CV diseases. ${ }^{9}$ Campos et al., ${ }^{1}$ found a high prevalence of cardiovascular risk factors in psoriatic arthritis patients, and the majority of

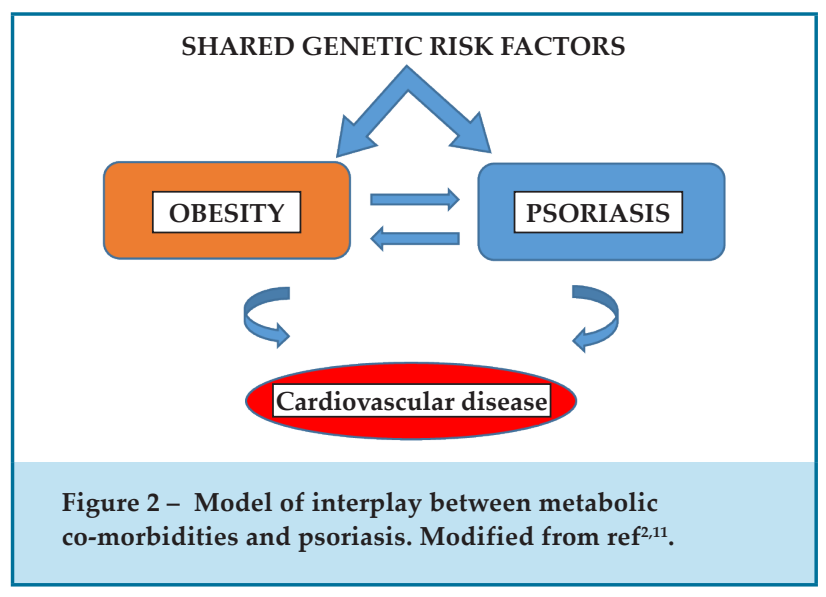

the sample was stratified into high or intermediate CV risk. This raises important issues regarding this association, including determining the minimum degree of psoriasis severity required to significantly increase the CV risk.

So, despite not being a (yet) consensual topic, there is strong evidence to suggest that psoriasis is an independent risk factor for the development of metabolic and CV comorbidities. In this regard, treatment of psoriasis might be expected to reduce the risk of developing these diseases. ${ }^{2,9}$ Although inflammation suppression, mainly by immunomodulatory agents, has been suggested as a promising target for the management of CV diseases, Campos et al., ${ }^{1}$ found no significant difference in $\mathrm{CV}$ parameters with the different drugs used by the study population. Nevertheless, other studies have shown positive effects with the new cytokine-targeted therapies, mainly anti-TNF $\alpha{ }^{8}$ A limitation of the study by Campos et al. ${ }^{1}$ is, in fact, its cross-sectional nature and absence of a control group.

In conclusion, since optimal treatment of psoriasis is the one that not only ameliorates skin condition but also decreases the risk for $\mathrm{CV}$ and metabolic disorders, an early diagnosis and appropriate intervention made by internal medicine physicians, dermatologists and immunologists, are mandatory. ${ }^{9}$ Campos et al.,${ }^{1}$ highlight the importance of assessing CV risk in psoriasis patients (mainly, but not exclusively with the severe forms of the disease), reinforcing this new perspective for inflammatory skin conditions. 


\section{References}

1. Campos BPS, Gomes GDS, Braz AS, Vilela AT. Cardiovascular Risk Factors and Risk Measurement in Patients with Psoriatic Arthritis in a University Hospital. Int J Cardiovasc Sci. 2020; 33(2):112-118.

2. Reich K. The concept of psoriasis as a systemic inflammation: implications for disease management. J Eur Acad Dermatl Venereol. 2012;26(Suppl 2):3-11

3. Haroon M, Gallagher, P.; Heffernan, E.; FitzGerald, O. High prevalence of metabolic syndrome and of insulin resistance in psoriatic arthritis is associated with the severity of underlying disease. J. Rheumatol. 2014;41(4):1357-65.

4. Boehncke WH, Boehncke S, Tobin AM, Kirby B. The 'psoriatic march': a econcept of how severe psoriasis may drive cardiovascular comorbidity. Exp Dermatol. 2011;20:303-7.

5. Yamanaka K, Mizutani H. "Inflammatory skin march": IL-1 mediated skin inflammation, atopic dermatitis, and psoriasis to cardiovascular diseases. J Allergy Clin Immunol. 2015;136(3):823-4

6. Fumian MM, de Motta NA, Maia R, Fraga CA, Barreiro EJ, de Brito FC. LASSBio-1425, an analog of thalidomide, decreases triglyceride and increases HDL cholesterol levels by inhibition of TNF-a production. Int J Cardiol. 2016 Jan 1;202:497-9.

7. Flammer AJ, Ruschitzka F. Psoriasis and atherosclerosis: two plaques, one syndrome? Eur Heart J. 2012;33:1989-91.

8. Furue, M.; Kadono, T. "Inflammatory skin march" in atopic dermatitis and psoriasis. Inflamm Res. 2017;66(10):833-42.

9. Hu SC, Lan CE. Psoriasis and cardiovascular comorbidities: focusing in severe vascular events, cardiovascular risk factors and implications for treatment. Int J Mol Sci 2017;18(10):2211.

10. Su YS, Yu HS, Li WC, Ko YC, Chen GS, Wu CS, et al. Psoriasis as initiator or amplifier of the systemic inflammatory march: impact on development of severe vascular events and implications for treatment strategy. J Eur Acad Dermatol Venereol. 2013;27(7):876-83.

11. Davidovici BB, Sattar N, Prinz JC, Puiz L, Emery P, Barker JN, et al Psoriasis and systemic inflammatory diseases: potential mechanistic links between skin disease and co-morbid conditions. J Invest Dermatol. 2010;130(7):1785-96. 